30.2\% ( $n=16)$ were current smokers, with a mean Medical Research Council (MRC) breathlessness grade 3) (table 1).

Of those assessed $48.15 \%(n=26)$ completed, of those who started $(n=36) 72.22 \%$ completed. Only the HADS anxiety domain demonstrated a clinically significant improvement in mean score post VPR (table 1). The COPD PREM 9 does not have an MCID, however completion of VPR lead to a mean improvement in score of 2.35 .

Conclusion Completion of an inner City London VPR programme did improve levels of anxiety and improved patient experience of living with disease, clinically significant improvements in exercise tolerance or symptom burden were not demonstrated. VPR provided a solution for delivering PR during the pandemic, however greater comparison against traditional face to face PR models is required to ensure it delivers the same healthcare and patient outcomes.

\section{P67 PILOT PROJECT: FEASIBILITY OF MINIMAL-EQUIPMENT HIGH-INTENSITY INTERVAL EXERCISE (HIIE) INTERVENTIONS IN BRONCHIECTASIS PATIENTS}

${ }^{1}$ KF Parrott, ${ }^{2} \mathrm{D}$ Taylor, ${ }^{3} \mathrm{~A}$ Jenkins, ${ }^{4} \mathrm{~A}$ Jones. ${ }^{1}$ United Lincolnshire NHS Trust, Lincoln, UK; ${ }^{2}$ University of Lincoln, Lincoln, UK; ${ }^{3}$ University of Nottingham, Nottingham, UK; ${ }^{4}$ Monash University, Melbourne, Australia

\subsection{6/thorax-2021-BTSabstracts. 177}

Introduction Minimal-equipment interval-type interventions offer a cost-effective way to improve the exercise adherence, fitness, and symptoms of bronchiectasis patients in the home setting. Of the approaches this might include, stair and walking-based high-intensity interval exercise (HIIE) have emerged as potentially safe, practical, and time-efficient methods for use by previously untrained and clinical populations in the home. However, the feasibility of using these methods to prescribe HIIE safely and effectively to bronchiectasis patients is yet to be explored.

Methods Following baseline physiological assessment (6MWT, CST, IQST) five non-cystic fibrosis bronchiectasis patients (2 men, 3 women, $68 \pm 5$ years) completed four differing step or walking-based HIIE formats in a randomised order across four supervised trials. HIIE sessions comprised of either $3 \mathrm{x}$ $20 \mathrm{sec}$ 'all out' stepping, 3 x $60 \mathrm{sec}$ 'vigorous' stepping, $3 \mathrm{x}$ $60 \mathrm{sec}$ 'all out' walking, or $3 \times 180 \mathrm{sec}$ 'vigorous' walking. Patients were then asked to complete 6 weeks of unsupervised home-based HIIE, choosing their preferred HIIE format on 3 days per week, after which baseline physiological assessments were repeated.

Results No adverse events were reported and all HIIE formats were tolerated by patients (mean heart rate and ventilation across formats: $78 \pm 5 \% \mathrm{HR}_{\max }$ and $35.4 \pm 10.0 \mathrm{~L} \cdot \mathrm{min}^{-1}$, respectively). Patients successfully completed $3.1 \pm 0.5$ sessions per week of unsupervised home-based HIIE. The most popular format was 3 x $60 \mathrm{sec}$ of 'all out' walking, which comprised $46 \%$ of all sessions completed. Paired samples ttests showed home-based HIIE significantly improved 6MWT $(483 \pm 79$ vs $574 \pm 58 \mathrm{~m}, \mathrm{p}<.05)$ and CST $(23 \pm 5$ vs 28 $\left.\pm 4 \mathrm{ml} \cdot \mathrm{kg} \cdot \mathrm{min}^{-1}, \mathrm{p}<.05\right)$ outcomes.

Conclusion This data suggests step and walk-based HIIE is feasible, safe, well tolerated and effective for bronchiectasis patients. The engagement and enjoyment reported by patients suggests HIIE may offer an effective approach for prescribing unsupervised exercise to bronchiectasis patients, particularly when such prescription incorporates different choices of HIIE format. We therefore plan to investigate the effectiveness of these approaches to HIIE in a fully powered clinical trial and subsequently compare the benefits to other commonly prescribed formats of exercise.

\section{P68 A RETROSPECTIVE SERVICE EVALUATION OF A VIRTUAL RESPIRATORY PHYSIOTHERAPY OUTPATIENT CLINICAL SERVICE}

S Parkin, A Long, I Forys, R Allen. St Bartholomew Hospital, London, UK

\subsection{6/thorax-2021-BTSabstracts. 178}

Aim To review the effectiveness of a virtual service delivery for Airway Clearance in a chronic respiratory population.

Methods A retrospective audit of virtual provision of Airway Clearance to a respiratory outpatient population. Patient's attendance and outcomes were reviews

Results 47 patients (36\% male, mean (SD) age 57 (16) were referred to the respiratory outpatient physiotherapy service for ACT between March 2020-2021.

$70 \%$ had a diagnosis of bronchiectasis. $87 \%$ attended appointments virtually with $74 \%$ via video call. To establish an effective ACT it took 2 appointments of 30 minutes. Figure 1 describes ACT taught. 5\% (2) of patients did not have English as their first language, requiring advocates to translate. Of those who attended virtually, 1 patient was admitted to hospital within 3 months of being discharged from the service with an infective exacerbation of bronchiectasis.

$6 \%$ (3) declined a virtual consultation with their main reason being access to technology. $60 \%$ (2) were male with an average age of 46 . Our DNA rate was $18 \%$.

Discussion Our findings indicate that virtual clinics are feasible for respiratory assessments and teaching an ACT. Patients could be taught different types of ACT within a few appointments, with no adverse events. The impact of the COVID pandemic resulted in changes to outpatient services with an immediate effect. Therefore patients had limited choice on their appointment type and so it is difficult to compare these outcomes to face to face clinics from previous years. This change in practice did overcome other historical barriers prior to the pandemic ie. Travel distance and cost and infection control. Admission rates may have been lower due to shielding, resulting in reduced exposure. They do not reflect exacerbations managed at home via GP's.

Further work is required to identify the long term carry over and effectiveness of these treatments. Qualitative data on patient's perceptions of a virtual clinic would guide any long term changes to the service.

Conclusion Virtual outpatient clinics for respiratory physiotherapy are feasible. The change in service design may

\begin{tabular}{lllll} 
Abstract P68 Figure 1 & & & \\
\hline & $\begin{array}{l}\text { total virtual and } \\
\text { telephone }\end{array}$ & $\%$ & virtual & telephone \\
\hline $\begin{array}{l}\text { Number taught } \\
\text { acapella }\end{array}$ & 24 & $58 \%$ & 22 & 2 \\
$\begin{array}{l}\text { Number taught ACBT } \\
\text { Number taught other }\end{array}$ & 16 & $40 \%$ & 12 & 4 \\
\hline
\end{tabular}


improve access to our service and indicates a potential path for digital transformation within respiratory outpatient physiotherapy.

\section{P69 DOES PATIENTS' EXPERIENCE IMPROVE ON THE PREM- 9 AFTER A 6-WEEK PULMONARY REHABILITATION PROGRAMME?}

SM Chavaillaz, EJ Chaplin, E Dayes, S Ward, SJ Singh, D Coope, L Houchen-Wolloff. Centre for Exercise and Rehabilitation Science, Pulmonary Rehabilitation Department, Leicester, UK

\subsection{6/thorax-2021-BTSabstracts.179}

Introduction Evidence suggests that PR has a huge impact on a number of outcomes for patients with Chronic Obstructive Pulmonary Disease (COPD). ${ }^{1}$ However, limited data exists that has measured patients' experience of COPD pre and post rehabilitation. This study aimed to quantify the impact of PR on the experience of COPD patients using the Patient Reported Experience Measure 9 (PREM-9). ${ }^{2}$ The PREM-9 is a questionnaire designed to help health care professionals (HCPs) learn more about the patient experience of living with COPD.

Methods Patients with COPD attending a 6 week PR programme in the United Kingdom consented to use their clinical data, including the PREM-9.

SPSS was used to analyse the change in PREM-9 total score, and individual items post PR. The change in PREM-9 was correlated with the change in other routinely collected data COPD Assessment Test (CAT), Hospital Anxiety and Depression Scale (HADS) and the Incremental Shuttle Walk Test (ISWT).

Results Data from 69 participants was analysed, 35 males, mean [SD] $\mathrm{FEV}_{1}$ (\%) 57.3\% [19.6], age 66 [10.2] years. A significant mean change was noted for the PREM-9 of -3.13 (95\%CI: -5.39 to -0.88 ). Change in individual PREM-9 questions and domains are reported in table 1.

There was a weak positive correlation between the PREM9 questionnaire change with the change in HADS anxiety domain $(r=0.35, p=0.04)$ and the change in CAT questionnaire $(r=0.38, p=0.02)$. However, no significant correlation

\begin{tabular}{lllll} 
Abstract P69 Table 1 & COPD PREM-9 results & & \\
\hline & & $\begin{array}{l}\text { Pre PR } \\
\text { Mean } \pm[S D]\end{array}$ & $\begin{array}{l}\text { Post PR } \\
\text { Mean } \pm[S D]\end{array}$ & P value \\
& & & \\
\hline PREM-9 & Question 1 & $2.79 \pm 1.5$ & $2.05 \pm 1.49$ & \\
My everyday life & Question 2 & $1.4 \pm 1.53$ & $1.5 \pm 1.25$ & \\
with COPD & Question 3 & $2.28 \pm 1.44$ & $1.72 \pm 1.2$ & \\
& Question 4 & $1.79 \pm 1.44$ & $1.37 \pm 1.27$ & \\
PREM-9 & Question 5 & $2.14 \pm 1.55$ & $1.84 \pm 1.27$ & \\
Usual care in COPD & Question 6 & $1.45 \pm 1.33$ & $1.17 \pm 1.01$ & \\
& Question 7 & $1.64 \pm 1.3$ & $1.02 \pm 0.87$ & \\
PREM-9 & Question 8 & $1.93 \pm 1.32$ & $1.67 \pm 1.44$ & \\
COPD exacerbation & Question 9 & $1.88 \pm 1.38$ & $1.7 \pm 1.39$ & \\
PREM-9 & Total score & $17.19 \pm 9.26$ & $14.05 \pm 7.76$ & $.008^{*}$ \\
HAD Anxiety & Total score & $8.54 \pm 5.43$ & $6.49 \pm 4.74$ & $.005^{*}$ \\
HAD Depression & Total score & $6.72 \pm 3.94$ & $5.7 \pm 4.09$ & .098 \\
CAT & Total score & $22.79 \pm 7.7$ & $19.43 \pm 6.31$ & $.003^{*}$ \\
ISWT & Total score & $291.71 \pm 149.33$ & $338.29 \pm 158.9$ & $.000^{*}$ \\
\hline * statistical difference $P=<0.05$ & & & \\
& & & &
\end{tabular}

was found between the PREM-9 change and the HADS depression domain or ISWT change.

Conclusion Patients' experience of living with COPD improved after a 6-week PR programme especially in coping with their COPD every day. These changes were associated with positive changes in anxiety and symptoms. Therefore, this questionnaire provides a unique insight into the patients' experience with their care and may act as a quality indicator for benchmarking the PR service.

Ethics Number: 17/EM/0156

\section{REFERENCES}

1. Spruit, et al. An official American Thoracic Society/European Respiratory Society statement: key concepts and advances in pulmonary rehabilitation. AJRCCM 2013;188(8):e13-e64.

2. Hodson., et al. Development and first validation of a patient-reported experience measure in chronic obstructive pulmonary disease (PREM-C9). Thorax 2019;74 (6):600-603.

\section{P70 THE IMPACT OF POST COVID-19 REHABILITATION ON HOSPITAL AND NON-HOSPITALISED PARTICIPANT- IS THERE A DIFFERENCE?}

${ }^{1} \mathrm{SJ}$ Singh, ${ }^{2} \mathrm{AC}$ Watt, ${ }^{2} \mathrm{~L}$ Houchen-Wolloff, ${ }^{2} \mathrm{~S}$ Ward, ${ }^{2} \mathrm{EJ}$ Chaplin, ${ }^{2} \mathrm{NY}$ Gardiner, ${ }^{2} \mathrm{E}$ Daynes, ${ }^{1}$ University of Leicester, Leicester, UK; ${ }^{2}$ University Hospitals of Leicester NHS Trust, Leicester, UK

\subsection{6/thorax-2021-BTSabstracts. 180}

Background There is evidence that those hospitalised with COVID-19 have a significant and persistent symptom burden. Early data suggests that symptoms and functional deficit may be favourably influenced by a structured rehabilitation programme. There is little data describing the impact of a rehabilitation programme on those who had COVID-19 but were not hospitalised and managed in the community who also have persistent burdensome symptoms. We therefore wanted to explore early outcome data from rehabilitation post infection and specifically explore data from those referred from the community to our rehabilitation programme.

Methods Participants were recruited to a 6 week out-patient supervised rehabilitation programme, with sessions twice a week. The programme was developed specifically for the post COVID-19 population using educational materials from the YourCovidRecovery website. The outcomes were: the incremental shuttle walking test (ISWT) (including a familiarisation test at baseline), the endurance shuttle walking test (ESWT), COPD Assessment Test (CAT), Functional Assessment of Chronic Illness Therapy Fatigue Scale (FACIT), Hospital Anxiety and Depression Scale (HADS), EuroQual 5 domains (EQ5D) thermometer, the Montreal Cognitive Assessment (MoCA) and quadriceps maximum voluntary contraction (QMVC) (chair based strain gauge). Data was analysed using SPSS v25. A paired/independent t-test was used to compare changes before and after rehabilitation between and within groups.

Results $\mathrm{N}=82$ individuals completed the COVID-19 rehabilitation programme (45 male, mean [SD] age 58.1(16.2) years), 49 white British. 62 individuals were admitted to hospital (11.8(14.8) days) and 20 non-hospitalised. Overall the data identified significant improvements in the whole group for the ISWT, ESWT HADS (D), CAT,FACIT, QMVC 